\title{
Sarcocystis hominis
}

National Cancer Institute

\section{Source}

National Cancer Institute. Sarcocystis hominis. NCI Thesaurus. Code C124385.

A species of Sarcocystis protozoa that uses cows as the intermediate host and humans as the definitive host. Infections with S. hominis are rare, are often asymptomatic, and generally clear spontaneously. 